\title{
Magnetoplasmadynamic (MPD) accelerator assisted synthesis of diamond*
}

\author{
J J Blandino ${ }^{1}$ and D G Goodwin ${ }^{2}$ \\ ${ }^{1}$ Mechanical Engineering Department, Worcester Polytechnic Institute, Worcester, MA \\ 01609, USA \\ ${ }^{2}$ Division of Engineering and Applied Science, California Institute of Technology, Pasadena, \\ CA 91125, USA
}

Received 7 January 2004

Published 4 November 2004

Online at stacks.iop.org/PSST/13/712

doi:10.1088/0963-0252/13/4/022

\begin{abstract}
The use of a magnetoplasmadynamic (MPD) accelerator for diamond synthesis is investigated. The acceleration process in an MPD device is reviewed and results presented for a test in which a low power $(15 \mathrm{~kW})$ MPD accelerator was used as the gas activation source for diamond deposition. A hydrogen-argon mixture was used in the discharge with externally injected methane as the carbon precursor. Results, including SEM images, Raman and x-ray diffraction spectra are presented for a $3.3 \mu \mathrm{m}$ thick film deposited over a 100 min period. The Raman spectra include a broad background and shift in the diamond peak, indicative of contamination and stresses in the deposited film. Potential scaling benefits of the MPD accelerator as well as some of the drawbacks associated with methane injection and sample contamination underscored by the experiments are identified and discussed.
\end{abstract}

\section{Introduction}

\subsection{The MPD accelerator}

In contrast to an arcjet which relies on recovery of electrothermal energy in a nozzle to accelerate a gas, the magnetoplasmadynamic (MPD) accelerator represents a class of devices in which electromagnetic forces are predominantly used to convert electrical energy into directed kinetic energy. Further distinctions are made as to whether the magnetic field is primarily due to an externally applied field or self-induced by the discharge current itself.

In a typical self-field device (figure 1), the gas to be accelerated is injected into the annulus between a co-axial pair of electrodes. A current, which can range from several hundred to over ten thousand amperes, is established between the electrodes. The central cathode is typically made of tungsten and operates at a temperature of approximately $3000^{\circ} \mathrm{C}$. Thermionic emission of electrons into the relatively low pressure annulus sustains the diffuse discharge.

The qualitative dependence of momentum flux (thrust) on current can in large part be explained through a simplified

* This work was performed at the Jet Propulsion Laboratory, California Institute of Technology, Pasadena, CA, USA.

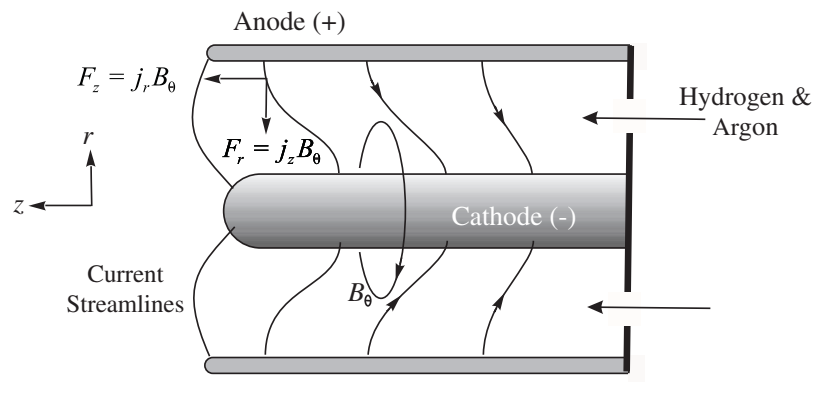

Figure 1. Self-field MPD accelerator.

physical model in which the Lorentz body force is integrated over the volume where acceleration takes place [1]. In this model, the axial components of the current lines produce an azimuthal magnetic field, which in turn interacts with the radial and axial components of the current to produce electromagnetic body forces. The axial and radial components of this body force are often referred to as the 'blowing' and 'pumping' components, respectively.

If used as a propulsive device (for spacecraft), the blowing contribution accounts for most of the thrust whereas the 
pumping component establishes a radial pressure distribution within the volume but does not contribute to thrust except at the cathode tip. Some limitations on the operating range of these devices have been observed over the last several decades during which they have been studied for potential use in spacecraft propulsion. For example, as the discharge current is increased for a given mass flow rate, the pumping force is believed to lead to a depletion of current carriers at the anode. Under these conditions, the discharge becomes unstable with a uniform current attachment transitioning to a spot attachment mode with very high localized current densities on both the anode and cathode. In addition to the spot attachment of the current, this transition is characterized by significantly increased cathode erosion rates, ablation of insulating surfaces and asymmetrical discharge with rapid voltage fluctuations with frequencies of hundreds of kilohertz and several megahertz [2]. For a given electrode geometry and gas, this transition, referred to as 'onset', occurs at a critical value of the parameter defined by the ratio of the current squared to the mass flow rate $J^{2} / \dot{m}$. Yoshikawa and Kagaya [3] have observed this transition in hydrogen at a value of $J^{2} / \dot{m}$ of $185 \mathrm{kA}^{2} \mathrm{sg}^{-1}$ which they compare to a theoretically predicted value of $266 \mathrm{kA}^{2} \mathrm{~s} \mathrm{~g}^{-1}$. For a given geometry, the $J^{2} / \dot{m}$ parameter also correlates with the mode of acceleration. An accelerator operating at a high value of $J^{2} / \dot{m}$ will typically be operating in an electromagnetic acceleration dominated regime, while a lower value of this parameter corresponds to a more electrothermal regime.

For a purely electromagnetic accelerator the thrust produced is found to scale as the square of the current (due to the $\vec{J} \times \vec{B}$ body force) and the ratio of the cathode to anode radii. A derivation of the well-known Maecker thrust equation can be found in the textbook by Jahn [1] for the case of a purely co-axial, self-field, electromagnetic accelerator:

$$
T=\dot{m} u_{\mathrm{e}}=\frac{\mu_{0}}{4 \pi}\left(\ln \frac{r_{\mathrm{a}}}{r_{\mathrm{c}}}+\frac{3}{4}\right) J^{2},
$$

where $r_{\mathrm{a}}$ and $r_{\mathrm{c}}$ are the anode and cathode radii, respectively. The thrust is the product of the mass flowrate $\dot{m}$ and a bulk jet velocity $u_{\mathrm{e}}$ and scales with the logarithm of the ratio of the radii. A more accurate, semi-empirical scaling relation has been developed by Choueiri, which captures the thrust dependence on mass flow rate as well as current [4]. The mean gas jet velocity (of more significance to material processing than the thrust) will scale as the ratio of the current squared divided by the mass flow rate $J^{2} / \dot{m}$, the same parameter that defines the beginning of the onset phenomenon. The mass flow rate required to produce a desired level of thrust will depend on the particular geometry since the pressure (and hence the number density of current carriers) must be sufficient to adequately sustain the required current. The geometry (cathode and anode length and radii) in turn is in large part determined by the need for the cathode to thermionically emit high current densities while operating below the melting point of tungsten. The difficulty in testing devices at power levels of megawatts has led to numerous studies using the so-called 'quasi-steady' devices. These are MPD thrusters driven by pulse forming networks capable of delivering tens of thousands of amperes over periods of several milliseconds. This time period is believed to be large compared with the timescales for the relevant plasma and gas kinetic phenomena but small compared to thermal time constants.

Most MPD devices use tungsten cathodes with added thorium (typically 2\%). This has the effect of lowering the work function improving thermionic emission and overall device efficiency. For space applications, it is likely that any MPD device would be radiation cooled. Most of the devices described in the literature, however, have used water-cooled copper anodes. From a practical standpoint, a water-cooled anode has distinct advantages in the laboratory as the thermal demands of radiation cooled tungsten anodes are significantly relaxed without altering the acceleration process.

While the MPD accelerator has been tested with many gases including argon, nitrogen, ammonia, and xenon, its use with hydrogen, which has the most relevance to materials processing applications, is considered here. The 'thrust efficiency' of an MPD device (defined as directed jet power produced divided by electrical power required) generally increases with power level. One reason for this is that the power losses in the electrode sheaths become a smaller fraction of the total power input. Attempts to increase thrust, particularly at power levels below $100 \mathrm{~kW}$, have often employed some form of magnetic field augmentation, usually through the use of electromagnets and, less frequently, through permanent magnets. In addition to enhancing the $\vec{J} \times \vec{B}$ body force for a given current, the applied magnetic fields help improve the uniformity of the discharge along the anode and extend the operational range of current before the critical onset value is reached. One study of such an 'applied field' MPD is that by Arakawa and Sasoh [5]. The work of Arakawa and others suggests the possibility that for materials processing applications, high jet velocity can be achieved at modest power levels $(<100 \mathrm{~kW})$ by operating at high values of $J^{2} / \dot{m}$ to ensure electromagnetic acceleration (within the onset limit) with the possible use of external magnetic fields to enhance acceleration. For a more complete review of hydrogen MPD work, the reader is referred to Blandino [6].

\subsection{Previous applications to CVD}

In 1994, Tanabe et al [7] published results on their use of an MPD for the deposition of what they referred to as hybrid amorphous carbons. These are amorphous carbons with mixed $\mathrm{sp}^{2}$ and $\mathrm{sp}^{3}$ bonding. These films were grown with a pulsed MPD device producing a 3-8 kA current pulse for approximately $1 \mathrm{~ms}$ (pulsed every $10 \mathrm{~s}$ ). The authors estimated a $50 \%$ degree of ionization $10 \mathrm{~cm}$ downstream of the exit plane at a chamber pressure of $2.2 \times 10^{-4}$ Torr. Substrate temperatures for these films varied between $25^{\circ} \mathrm{C}$ and $600^{\circ} \mathrm{C}$. Gases used for these tests included hydrogen and methane as well as oxygen and carbon tetrafluoride injected directly into the discharge. The authors stated that '.. no studies on synthesizing films using the MPD arc-jet have ever been reported, probably because it was developed as a thruster and not a film generator.' But, in 1991, Norton Co. had reported [8] that it had acquired the rights to a magnetoplasmadynamic arc (MPDARC) source for use in diamond film production, although to the best of the authors' knowledge no results of any tests have been published.

Blandino [6] has investigated some of the issues associated with the use of the MPD device for diamond deposition. One of 


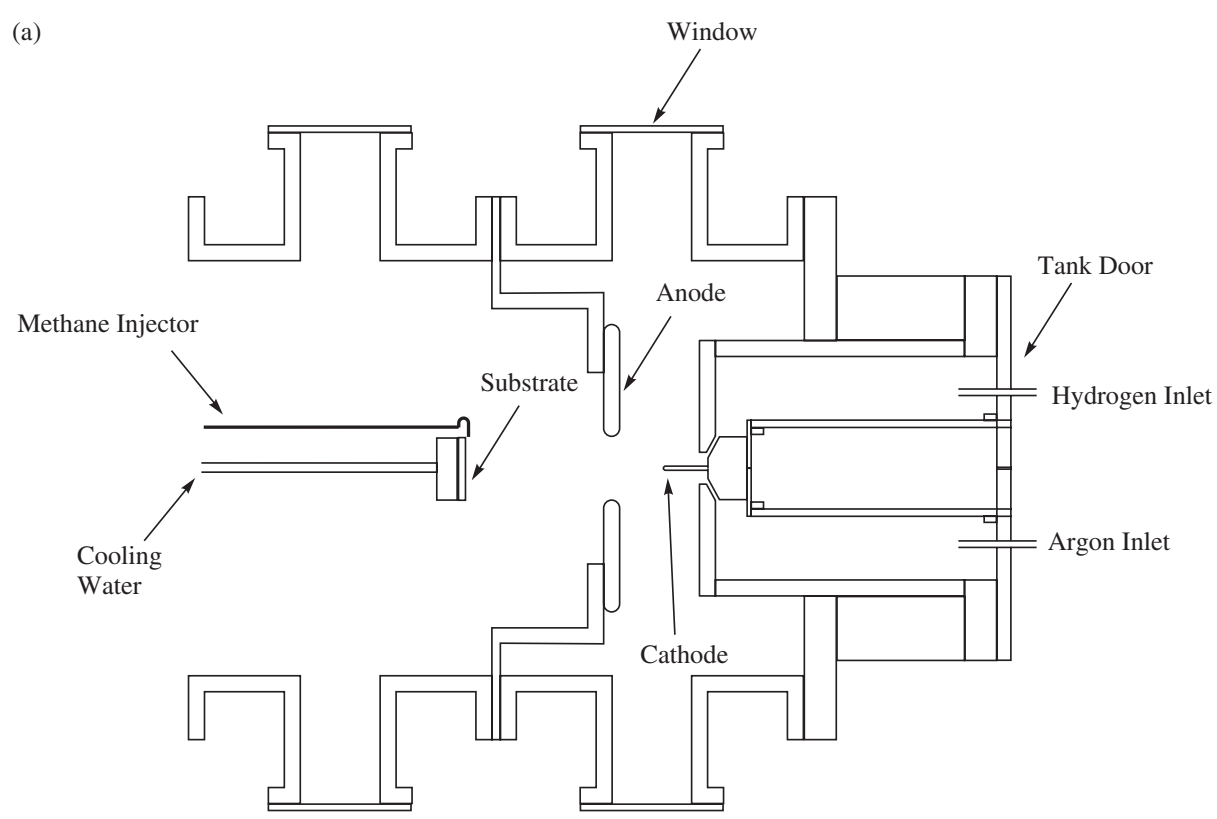

(b)

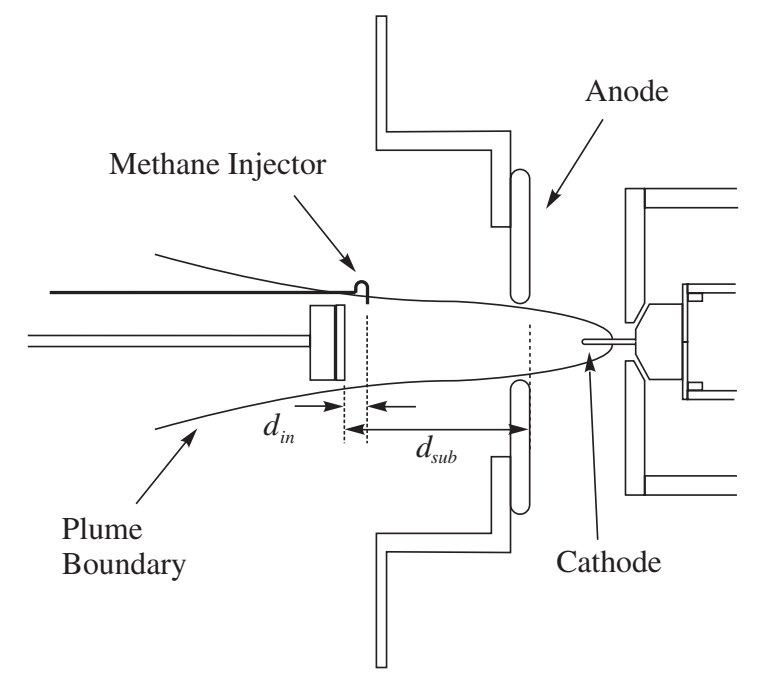

Figure 2. (a) Cathode test facility at JPL used for MPD assisted diamond film deposition studies and $(b)$ internal layout showing anode, cathode, substrate and methane injector.

the possibilities considered was the use of a biased substrate to heat electrons in the boundary layer and enhance dissociation. An energy balance was used to determine an upper bound on the level of electron heating obtainable for a given bias current density. Results showed that even for pressures of a few Torr and ionization fractions of 5-25\%, the required current for a few thousand degrees increase in electron temperature over the heavy particle gas temperature was of the order of several tens of $\mathrm{A} \mathrm{cm}^{-2}$. From that analysis it was concluded that high plasma conductivity in an MPD plume and electron energy losses through inelastic molecular collisions would preclude the effective use of Ohmic heating of the electrons as a means of enhancing electron catalysed dissociation in the boundary layer.

Based on both the experimental and analytical investigations of the MPD source for diamond deposition reported in [6], the potential for higher growth rates than those obtainable with more conventional plasma sources was not found to be significant enough to offset the disadvantages associated with contamination of the film due to metal vapour from the high current electrodes and poor entrainment of the carbon precursor gas due to the short residence time in the plume. This paper reports results from one of these tests performed using a low power MPD device for diamond deposition.

\section{Experiment}

The facility used for this work was originally set up at NASA's Jet Propulsion Laboratory for the purpose of investigating the lifetime and thermal behaviour of high current, thermionic cathodes such as those used in MPD (or Lorentz Force Accelerator) thrusters for space propulsion. A diagram of the vacuum chamber for this facility is shown in figure 2 . 

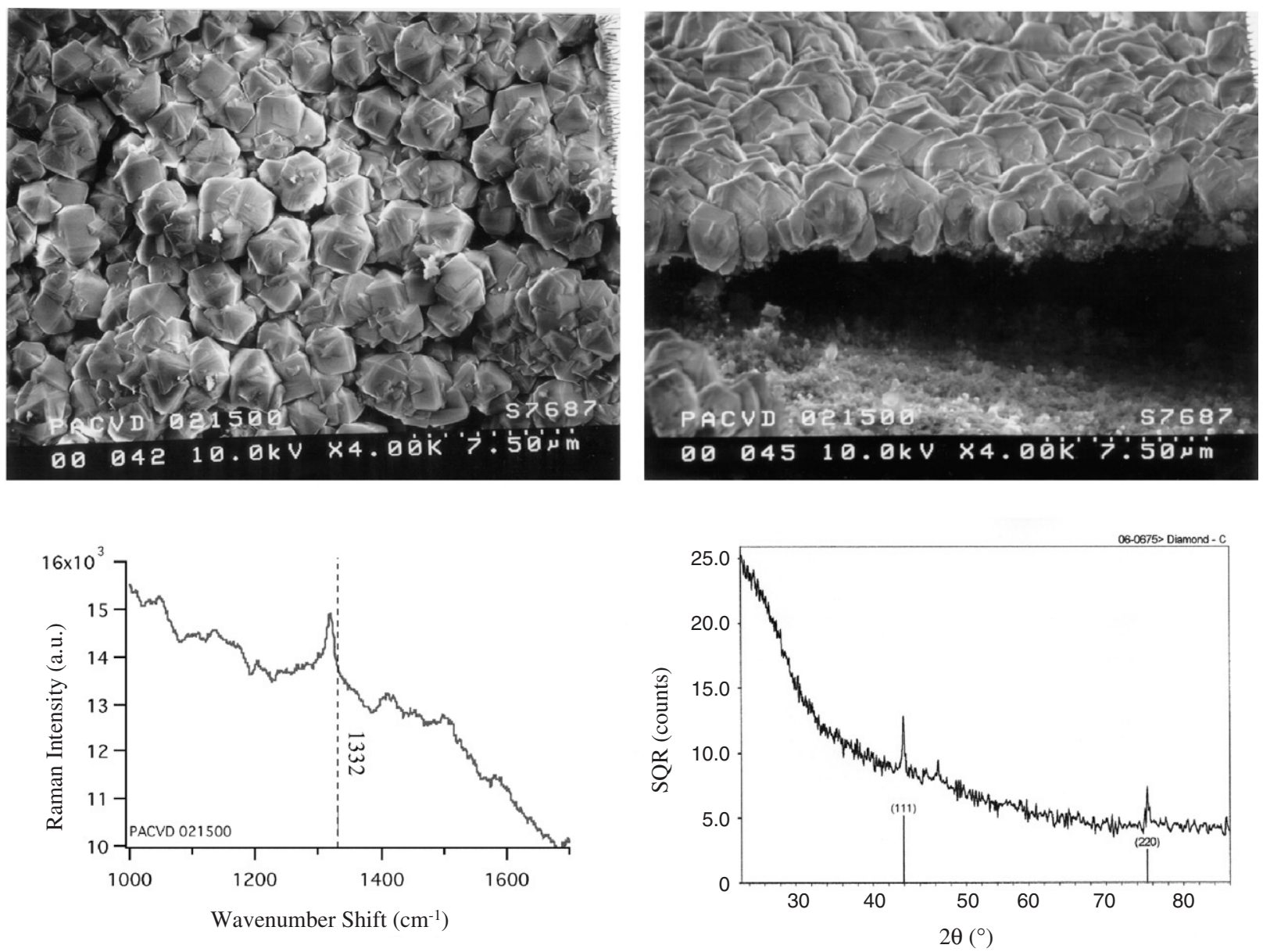

Figure 3. SEM image (top and edge), Raman and XRD spectra for MPD assisted film deposition: Meth/Hyd $=1.6 \%, d_{\text {in }}=0.5 \mathrm{~cm}$; injector located on side, as shown in figure 2 .

The chamber is approximately $0.5 \mathrm{~m}$ in diameter and $2.4 \mathrm{~m}$ long. As shown in the diagram, it consists of cylindrical segments which are bolted together with O-ring seals. A number of ports on these segments facilitate electrical and water feed, optical access to the area where the substrate is located, as well as access to the cathode. The chamber is pumped by a 610 litre $\mathrm{s}^{-1}$ Roots blower backed by a 140 litre $^{-1}$ mechanical pump. This pumping combination was originally selected for this facility to enable sustained pumping of relatively high flow rates used in the cathode tests $\left(>0.3 \mathrm{~g} \mathrm{~s}^{-1}\right.$ of argon). Because the efficiency of a blower decreases in the milli-Torr range, the ultimate pressure for this facility was only 10-20 mTorr (1.3-2.7 Pa).

Figure 2(b) shows the relative location of the water cooled substrate, anode, and cathode within the chamber along with an approximate outline of the visible portion of the plume jet. The cathode is a $0.95 \mathrm{~cm}$ diameter, $7.6 \mathrm{~cm}$ long thoriated tungsten rod mounted in a water cooled copper mount. The cathode is mounted on the tank door, which slides away from the end of the tank on rails for access. The anode consists of a separate, water-cooled copper ring supported by a water cooled cylinder supported between the first two tank segments. The anode opening is $7.6 \mathrm{~cm}$ in diameter. As evident from the open electrode design (as compared with figure 1, for example), this particular configuration was originally designed for easy optical access to the cathode and not for high efficiency acceleration. The MPD thruster design is a derivative of the segmented anode 'DT' MPD thruster series built and operated at Stuttgart [9]. In operation, an arc is established by applying a high voltage between the electrodes once gas flow has been started. An $850 \mathrm{~V}, 4 \mathrm{~A}$ start supply is used to initiate the arc, which is then sustained by one of two available Miller $1500 \mathrm{~A}$, $40 \mathrm{~V}$ power supplies. This arrangement enables operation up to $120 \mathrm{~kW}$ total power. For the diamond deposition studies reported here, operation was limited to 700-950 A at a discharge voltage of approximately $18 \mathrm{~V}$ (total power 13$17 \mathrm{~kW}$ ) and was limited primarily by heat flux to the substrate as well as the anode.

No plume velocity measurements have been made with this device, but an upper bound on the gas velocity can be inferred from a review of tests at the Stuttgart facility at similar argon flowrates and operating current. The contoured, segmented anode of the Stuttgart facility would be expected to provide much more efficient electromagnetic acceleration so the performance at a current of $900 \mathrm{~A}$ at an argon flow rate of $0.8 \mathrm{~g} \mathrm{~s}^{-1}$ provides a reasonable upper bound on jet velocity in the JPL facility. From figure 3 in Wegmann et al [9], the velocity can be estimated to be approximately 2500 $3000 \mathrm{~m} \mathrm{~s}^{-1}$. This velocity is at least a factor of $\frac{1}{3}$ lower than the jet velocity of an arcjet expanding supersonically into low pressure. The electrode design and relatively low 
current $(<1 \mathrm{kA})$ imposes a significant limitation on the velocity achievable in the facility used here.

The substrates used for these tests consisted of a molybdenum disc, $7.0 \mathrm{~cm}$ in diameter and $0.32 \mathrm{~cm}$ thick. The molybdenum disc was mounted with four screws to a water cooled copper support. Substrate temperature was inferred from Type-K thermocouples installed on the back surface of the substrate. Three thermocouples were used to measure substrate temperature from behind at the substrate centre and at two points close to the edge of the disc. Originally, the thermocouples were fixed to spring loaded contacts, recessed into the copper block, which would ensure contact with the back of the disc. With repeated use, the spring mechanism became less reliable and a new approach was adopted in which thermocouple beads, approximately $1 \mathrm{~mm}$ in diameter, were inserted into blind holes on the back of the molybdenum disc. These blind holes extended approximately $1-2 \mathrm{~mm}$ into the substrate. Different approaches to control substrate temperature were evaluated including the use of a separate coolant loop using a mixture of water and ethylene glycol with a dedicated chiller. The cooling capacity of this system proved to be insufficient so a facility water cooled by a chiller, which serves several labs, was used instead. The large thermal mass of this system enabled operation without any significant increase in supply-side water temperature. For the test, methane was used as the carbon precursor gas with a hydrogen/argon mixture used in the discharge. Figure 2 shows the location of the methane injection tube used in this test. Before the test, the molybdenum substrate was polished with $0.25 \mu \mathrm{m}$ diamond paste to facilitate nucleation.

As previously mentioned, the jet velocity expected from the MPD used was lower than an arcjet and certainly any MPD source that would be developed for use as a space thruster. The value of $J^{2} / \dot{m}$ in these tests was $1.3 \mathrm{kA}^{2} \mathrm{sg}^{-1}$, about two orders of magnitude lower than that of previous MPD work using hydrogen as mentioned in the introduction. As a result, it is unrealistic to expect that the facility available for these tests would be representative of MPDs, in general, at least with respect to benefits one might expect due to high jet velocity (and consequently thin boundary layers with sharp species concentration gradients). Nevertheless, one can expect that results of tests in this facility will provide some insight into issues associated with a low pressure, high current discharge, which results in higher levels of dissociation and ionization than one would expect in an arcjet operating near atmospheric pressure.

For this test, the discharge was operated with a nominal mixture of $23 \mathrm{slm}$ argon $\left(0.7 \mathrm{~g} \mathrm{~s}^{-1}\right)$ with $0.9 \mathrm{slm}$ hydrogen $\left(1.3 \mathrm{mg} \mathrm{s}^{-1}\right)$. Hydrogen results in a higher potential fall through the anode sheath resulting in higher levels of energy deposition and anode heating than argon. The mixture, therefore, serves to lower the heat load to the anode as well as facilitating arc initiation. Other groups have successfully employed argon/hydrogen discharges in diamond CVD and it is expected that the presence of argon contributes to the chemistry only through third-body collision effects.

A large uncertainty in the substrate temperature during the test was due to the significant variations of temperature, greater than $100^{\circ} \mathrm{C}$ in some cases, from one thermocouple to another suggesting nonuniform heat flux to the surface. This was, in part, probably due to the fact that the substrate was almost as wide ( $7 \mathrm{~cm}$ in diameter) as the plasma jet itself. Another factor contributing to the nonuniformity of heat flux to the surface was the fact that the plume was not centrally aligned in the chamber and the ability to position the substrate within the plume was limited.

In deposition experiments using supersonic arcjets, methane injection occurs very near the exit plane of the divergent nozzle as in the case of Loh and Cappelli [10,11]. In initial tests, the location used for methane injection was just downstream of the anode, approximately $14 \mathrm{~cm}$ from the substrate surface. The methane was injected from a ring with many small holes along its circumference, surrounding the plume. The tests performed with this injector ring consistently produced amorphous sooty deposits on the substrate.

Estimates were made by Blandino [6] of the optimal residence time for methane decomposition in the plume in order to maximize the flux of methyl radicals and atomic hydrogen to the substrate-two species that have been identified as playing a significant role in high rate, high quality diamond synthesis. At pressures of 100 and $333 \mathrm{~Pa}$, which approximate the pressure in the plume and stagnation regions, there is little recombination of the hydrogen even at a temperature of $5000 \mathrm{~K}$, which one could expect well into the thermal boundary layer for an MPD stagnation flow. The methyl mole fraction reaches a maximum at a residence time of 1-3 $\mu \mathrm{s}$. Achieving adequate entrainment and mixing of the methane in the hydrogen jet on such a short timescale is a very difficult challenge.

For the ring injector configuration, even if one assumes the methane is fully entrained in the plasma jet and travels with a velocity of $2000 \mathrm{~m} \mathrm{~s}^{-1}$, the relatively large distance of the injector from the substrate results in a residence time of $70 \mu \mathrm{s}$. With such a relatively long time for decomposition to occur, the mole fraction of atomic carbon is sufficiently high to produce significant amounts of amorphous deposits. As a result of these initial tests, the remainder of the testing included an injector mounted to the substrate support with a single tube adjacent to the substrate surface. After some unsuccessful attempts at using alumina injection tubes, a design using $1.6 \mathrm{~mm}$ (outer diameter) molybdenum proved effective. The diameter of the visible plume at the substrate position was at least $8-10 \mathrm{~cm}$. As a result, the injector tube was subject to significant heat loads even when located off to the side as in figure $2(b)$. In this side location, there was some carbon build-up on the injector and also on the region of the substrate closest to the injector although not nearly as extensive as observed in some tests with the injector mounted over the centre of the substrate. This is probably due to the fact that the injector temperature was lower since it was out of the core flow.

The Raman spectrometer used for this work was a HoloProbe Raman System manufactured by Kaiser Optical Systems Inc. A $785 \mathrm{~nm}$ laser with a power level of $50 \mathrm{~mW}$ was used and is focused through a microscope onto the sample, enabling a spatial resolution of $0.5-2.0 \mu \mathrm{m}$.

\section{Results}

Figure 3 presents results for a test performed under the conditions listed in table 1 resulting in a deposited film that was 
Table 1. Test conditions and results for deposition (estimated growth rate assumes negligible time for nucleation).

\begin{tabular}{ll}
\hline Methane/hydrogen ratio $(\%$ by vol $)$ & 1.6 \\
Injector substrate distance $d_{\text {in }}(\mathrm{cm})$ & 0.5 \\
Anode-substrate distance $d_{\text {sub }}(\mathrm{cm})$ & 16.2 \\
Deposition time $(\min )$ & 100 \\
Substrate temperature $\left({ }^{\circ} \mathrm{C}\right)$ & $500-648$ \\
Discharge power $(\mathrm{kW})$ & 15.4 \\
Estimated growth rate $\left(\mu \mathrm{m} \mathrm{h}^{-1}\right)$ & 2 \\
\hline
\end{tabular}

several square millimetres in size. The substrate temperature range listed in table $1\left(500-648^{\circ} \mathrm{C}\right)$ represents the spatial variation across the substrate as characterized by the (timeaveraged) readings on each of the three thermocouples as described earlier. The higher value corresponds to the timeaveraged reading from one of the thermocouples close to the substrate edge. This same thermocouple also recorded the peak temperature of $698^{\circ} \mathrm{C}$ during the experiment. A SEM image of the film morphology is shown in the top two panels, including an edge view from which the film thickness was determined. Also included in the figure is the Raman spectrum taken at approximately the same location as the SEM images as well as the $\mathrm{X}$-ray diffraction (XRD) spectra.

The triangular faceting characteristic of a (111) oriented plane appears to be the most prevalent, which is supported by the XRD spectra. While a minor Raman peak at $1319 \mathrm{~cm}^{-1}$ is evident, the $1332 \mathrm{~cm}^{-1}$ peak characteristic of $\mathrm{sp}^{3}$ bonded diamond is absent. In addition, the spectrum displays a broader background, possibly due to luminescence of the samples. Two possible explanations for the poor Raman spectra of the sample is incorporation of nitrogen and a high level of defects localized along the grain and twin boundaries.

The potential effect of nitrogen contamination has been documented in a study by Vandevelde et al [12], characterizing the effect of different levels of nitrogen incorporation, one of the most common impurities in diamond. While relatively low levels of nitrogen in the gas phase $(<100 \mathrm{ppm})$ can contribute to higher quality structures, a high nitrogen concentration in the gas phase results in films with a higher degree of graphitic and amorphous carbon [12]. In the Vandevelde study, nitrogen concentrations in the gas stream were increased from 95 to $714 \mathrm{ppm}$. This increase resulted in a decrease in both the sharpness and intensity of the $1332 \mathrm{~cm}^{-1}$ Raman peak. In addition to its effect on the characteristic Raman line, addition of nitrogen was also found to result in a preferred (100) orientation in the film morphology.

It is probable that nitrogen present in the gas stream during deposition played some role in the overall poor quality of the film as evident in the Raman spectra. Despite numerous attempts to eliminate all gas leaks in the chamber used for deposition, it is estimated that the background residual partial pressure of air could have been as high as 10 mTorr. For a nominal operating chamber pressure of 8.5 Torr, this corresponds to a nitrogen concentration of approximately $930 \mathrm{ppm}$. Based on the Vandevelde study, such a high concentration could easily account for the suppression of $\mathrm{sp}^{3}$ bonded carbon. The identification of the sample as diamond is further supported by the XRD spectra in which two peaks associated with diamond are evident.

\section{Conclusions}

\subsection{Potential advantages}

Three characteristics, in particular, suggest the possibility that one could obtain important enhancements in diamond film deposition using an MPD device. These are (1) the inherently higher level of dissociation and ionization of the gas species in the plume core, (2) the higher jet velocity, and (3) the scalability to larger deposition areas. Because MPD discharges utilize electromagnetic forces to achieve plasma acceleration, they are not limited by thermal constraints at a physical nozzle throat. For this reason, they are capable of operation at significantly higher power densities than arcjets. One consequence of operating at a higher power (and lower pressure) is the production of a high enthalpy plume with significant dissociation and ionization. Higher levels of dissociation will increase the number density of species required for growth of diamond and suppression of graphite. While dissociation of the hydrocarbon precursor gas and the hydrogen diluent gas is essential, it is not clear whether the higher ionization level will prove beneficial.

In an electromagnetic thruster, the thrust level scales approximately with the square of the current which is 1-2 orders of magnitude higher than the arcjet. As a result the mean gas velocities in the plume of a conventional MPD are expected to be higher than the arcjet. The consequence of higher jet velocity will be a higher stagnation temperature and thinner stagnation boundary layer at the substrate surface. The thinner boundary layer should result in sharper concentration gradients over the growth region and a higher flux of activated radicals to the surface.

Because the MPD is an electromagnetic device, it is more readily scaled up to higher power and/or larger electrodes to achieve deposition over a larger region of the substrate than a thermal arcjet which requires a nozzle. As evident from equation (1.1) electrode geometry can be scaled to maintain a manageable cathode current density while increasing the overall power and exhaust plume cross-sectional area. Such a scale-up could have significant commercial potential if the overall chemistry of the MPD plume is found to be effective for achieving growth.

\subsection{Potential disadvantages}

Some issues associated with substrate and methane injector heating became apparent during these studies. The severe heat fluxes associated with the MPD plume, even at low jet velocities, requires either very effective active cooling of the substrate or increasing its distance from the exit plane. Increasing the distance results in increased residence time for methane pyrolysis if the methane injection port is located at the MPD exit plane. This was overcome by moving the injector closer to the substrate. As a result, however, excessive heating at the injector opening resulted in high rates of amorphous carbon deposition at the tip with subsequent flow disruption. Locating the injector completely out of the plume increases the time needed for effective entrainment and diffusion across the plume (and also increases the time during which pyrolysis can occur). Locating it closer to the substrate as in the 
present experiment can lead to excessive injector heating, soot formation and flow disruption.

Because the MPD cathodes operate near their melting point, cathode erosion and subsequent contamination of the deposited film with tungsten vapour is inevitable. This effect was evident in other test cases described by Blandino [6] in which tungsten carbide was detected in the XRD spectra of some deposited samples. The discharge current in these other tests (approximately 845-860 A) was comparable to the current in the test reported here $(877 \mathrm{~A})$ in which no tungsten carbide was observed in the XRD spectra. This suggests better diagnostics, such as cathode temperature, might be needed to predict the onset of contamination from tungsten vapour.

In summary, while higher growth rates and scale up to larger deposition areas is a possibility, the quality of deposited films may limit their use to applications with less demanding requirements with respect to film quality.

\section{Acknowledgments}

This work was supported by the National Aeronautics and Space Administration's Advanced Propulsion Concepts Program. The authors would like to thank Drs Keith Goodfellow and James Polk, as well as Mr Al Owens and Mr Robert Toomath of the Jet Propulsion Laboratory for their assistance with the MPD facility.

\section{References}

[1] Jahn R 1968 Physics of Electric Propulsion (New York: McGraw-Hill)

[2] Merke W D, Auweter-Kurtz M, Habiger H, Kurtz H and Schrade H O 1988 AIAA 88-028, 20th DGLR/AIAA/JSASS Int. Electric Propulsion Conf. (Garmisch-Partenkirchen, W. Germany, 3-6 October 1988)

[3] Yoshikawa T and Kagaya Y 1982 Thrust and efficiency of new K-III MPD thruster AIAA 82-1887, 16th AIAA/JSASS/DGLR Int. Electric Propulsion Conf. (New Orleans, LA, 17-19 November 1982)

[4] Choueiri E 1998 J. Propulsion Power 14744

[5] Arakawa Y and Sasoh A 1988 J. Propulsion Power 4287

[6] Blandino J 2001 PhD Thesis California Institute of Technology

[7] Tanabe Y, Burkhard G, Ishikura T, Tsunoda K, Hasuo H, Tamaru M, Tamura H and Sawaoka A and Uematsu K 1994 Japan. J. Appl. Phys. Part 1-Regular Papers Short Notes and Review Papers 336684

[8] Manufacturing Brief 1991 Ceram. Bull. 70205

[9] Wegmann T, Auweter-Kurtz M, Habiger H A, Kurtz H L and Schrade H O 1992 Experimental investigation of steady state high power MPD thrusters AIAA 92-3464 28th Joint Propulsion Conf. (Nashville TN, 6-8 July 1992)

[10] Loh M H and Cappelli M A 1992 AIAA 92-3534, 28th AIAA Joint Propulsion Conf. (Nashville TN, USA, 6-8 July 1992)

[11] Loh M H and Cappelli M A 1993 Diamond Relat. Mater. 2454

[12] Vandevelde T, Nesladek M, Meykens K, Quaeyhaegens C, Stals L M, Gouzman I and Hoffman A 1988 Diamond Relat. Mater. 7152 